\title{
ЦИФРОВОЙ КЕРН: МОДЕЛИРОВАНИЕ АКУСТИЧЕСКОЙ ЭМИССИИ В ЦЕЛЯХ ЛОКАЛИЗАЦИИ ЕЁ ИСТОЧНИКОВ МЕТОДОМ ОБРАЩЕНИЯ ВОЛНОВОГО ПОЛЯ В ОБРАТНОМ ВРЕМЕНИ
}

Решетова Г. В., Анчугов А. В.

\begin{abstract}
Аннотация
В работе представлены результаты численных экспериментов для решения задачи локализации событий акустической эмиссии в образцах керна. В качестве математической модели использовались уравнения динамической теории упругости в полярной системе координат. Предложен подход к локализации событий акустической эмиссии методом обращения времени, основанный на анализе суммарной энергии волнового процесса. Параметры изучаемых процессов получены на основе обработки данных лабораторных экспериментов. Приведены результаты численного моделирования, на основании которых определяется ряд основных характеристик конфигурации многоканальной системы сбора данных: оптимальное количество каналов регистрации, расположение датчиков относительно керна. Исследована разрешающая способность метода в зависимости от доминирующей частоты и разного количества каналах регистрации.
\end{abstract}

\section{Ключевые слова:}

керн, геомеханические испытания керна, акустическая эмиссия, численное моделирование, продолжение волнового поля в обратном времени 


\title{
ЦИФРОВОЙ КЕРН: МОДЕЛИРОВАНИЕ АКУСТИЧЕСКОЙ ЭМИССИИ В ЦЕЛЯХ ЛОКАЛИЗАЦИИ ЕЁ ИСТОЧНИКОВ МЕТОДОМ ОБРАЩЕНИЯ ВОЛНОВОГО ПОЛЯ В ОБРАТНОМ ВРЕМЕНИ
}

\author{
Г.В. Решетова ${ }^{1}$, А.В. Анчугов ${ }^{2}$ \\ ${ }^{1}$ Институт вычислительной математики и математической геофизики СО РАН, 630090, \\ Россия, г. Новосибирск, пр. Лаврентьева, 6. \\ ²АО Геологика, 630055, Россия, г. Новосибирск, ул. Мусы Джалиля, 23.
}

\section{ВВЕДЕНИЕ}

Акустическая эмиссия (АЭ) является надёжным свидетельством процессов разрушения в твёрдом упругом теле и лежит в основе разнообразных методов неразрушающего контроля целостности объектов [Grosse and Ohtsu, 2008; Ohtsu, 2015]. AE возникает вследствие спонтанного выделения накопленной в некотором объекте энергии деформаций в результате необратимых процессов разрушения, обусловленных внешними воздействиями. Выделение энергии действует как источник возбуждения упругих волн, которые могут быть зарегистрированы на поверхности изучаемого объекта и подвергнуты анализу.

Методы контроля целостности объекта, основанные на акустической эмиссии, применяются с 1950 года. Начало этого направления исследований положено Джозефом Кайзером в его диссертации [Kaiser, 1950]. В настоящее время акусто-эмиссионный метод успешно используют для оценки технического состояния различных промышленных объектов. Так, например, известны созданные на этой основе способы контроля состояния парогазовых турбин, контроля целостности деталей системы охлаждения ядерных реакторов, контроля герметичности нефтегазовых трубопроводов [Кретов, 2014; ШайкоШайковский и др., 2016; Иванов и Барат, 2017] и множество других. Все эти подходы позволяют провести неразрушающую техническую диагностику объекта и оценить степень опасности возникновения дефектов и разрушений в реальном времени.

В нефтегазовой отрасли методы АЭ применяются для диагностики напряженнодеформированного состояния горных пород и изучения реакции насыщенных сред на внешние механические воздействия [Кочарян, Остапчук, 2015; Шадрин, 2016; Иголкина, Дрягин, 2018], для изучения свойств кернового материала в лабораторных условиях, в частности для изучению акусто-эмиссионного эффекта памяти при нагружении образцов горных пород [Шкуратник и др., 2004; Шкуратник, Новиков, 2015; Щербаков, Чмель, 2016; Дрягин, Володин, 2017]. Преимущество этого метода состоит в возможности проведения неразрушающего контроля образца керна за один цикл нагружения, при этом не требуется дорогой и трудоемкой процедуры томографического сканирования всего 
объекта. Достаточно специальным образом расположить датчики на поверхности керна и записать колебания, обусловленные процессами разрушения внутри образца. Эти колебания порождаются появлением и развитием микро- и макротрещин, схлопыванием микропор, трением уже существующих и возникающих новых поверхностей разлома друг о друга и другими локальными динамическими перестройками структуры образца. Принцип исследования остается тем же: образец горной породы подвергается различным механическим воздействиям. В результате такого воздействия, в образце возникает напряженно-деформированное состояние, сопровождаемое частичным или полным разрушением, что и приводит к акустической эмиссии.

Всё большее распространение методов АЭ для изучения керна обусловлено появлением современных установок для лабораторных исследований, снабженных многоканальными системами регистрации. В настоящее время в мире существует несколько типов такого оборудования, среди которых можно выделить MTS TerraTek, которое, по желанию заказчика, комплектуется системой регистрации АЭ. К сожалению, на российском рынке пока нет доступного отечественного оборудования аналогичного типа.

Данная работа ставит перед собой две цели:

1) Выполнить анализ параметров, которые должны быть заложены при создании отечественной измерительной системы, нацеленной на сбор данных акустической эмиссии;

2) Разработать численные методы и создать прототип программного обеспечения для моделирования процесса АЭ на реальных образцах керна стандартного размера.

Результаты моделирования, подкрепленные экспериментальными данными, будут использованы при разработке усовершенствованной установки для изучения прочностных свойств керна (УИДК) в пластовых условиях производства АО «Геологика» (Рис.1) в виде специализированного программного обеспечения по восстановлению событий АЭ в испытуемом образце. Здесь необходимо подчеркнуть, что единственной известной нам в настоящее время версией программного обеспечения, поставляемого на рынок, является InSite AE/Microseismic Software английской компании Itasca Consulting Ltd. Однако используемый в этом пакете математический аппарат не разглашается, что делает невозможным проверить достоверность восстановления событий АЭ этим программным продуктом и обеспечить его дальнейшее совершенствование в направлении повышения разрешающей способности и информативности.

Исходя из целей исследования, изложение материала разделено на три части. Первая часть содержит описание организации сбора данных в ходе лабораторных экспериментов. Далее следует раздел с описанием математической постановки задачи и методом математического моделирования АЭ. В третьей части приведены результаты численных экспериментов по восстановлению событий АЭ и их обсуждение.

\section{ВЫБОР ТЕХНИЧЕСКИХ ПАРАМЕТРОВ ПЕРСПЕКТИВНОЙ УСТАНОВКИ ДЛЯ ИЗУЧЕНИЯ АКУСТИЧЕСКОЙ ЭМИССИИ В ОБРАЗЦАХ КЕРНА}


Как уже было отмечено во введении, за основу разрабатываемого перспективного оборудования для изучения акустической эмиссии в образцах керна было решено взять установку УИДК производства АО Геологика, представленную на Рис.1. Она позволяет моделировать условия залегания керна горной породы как по температуре, так и по давлению, а также выполнять фильтрационные исследования с прокачкой различных флюидов. При проведении лабораторных исследований кернов на этой установке регистрация сигналов АЭ, как правило, не производится, однако все такие установки оснащены активной акустикой для измерения времени прохождения сигналов сквозь керн. Таким образом, уже существующий вариант оборудования имеет принципиальную возможность записывать данные акустической эмиссии. Для того, чтобы эти данные можно было использовать в дальнейшем нами и запланированы дополнительные исследования, базирующиеся как на усовершенствовании методов сбора и обработки реальных данных, так и на развитии методов математического моделирования процесса АЭ. Именно по результатам этого анализа и будет рекомендована конфигурация многоканальной системы. Регистрация сигналов АЭ позволит получить дополнительную информацию об объекте исследования при проведении лабораторных испытаний. В частности, даст возможность в реальном времени наблюдать за поведением материала при нагружении и контролировать процессы его деформации и разрушения, прогнозировать изменение напряжённого состояния на ближайшее время (миллисекунды) и корректировать, при необходимости, ход эксперимента.

Сбор данных. Изначально сбор данных АЭ был реализован с целью изучения эффекта Кайзера [Bai et al.,2018]. Напомним, что этот эффект заключающегося в том, что при испытаниях образцов в режиме повторяющихся нагрузок и разгрузок акустическая эмиссия при повторном нагружении наблюдается только с того момента, когда напряжение превышает максимальную величину, достигнутую при предыдущем нагружении. Однако по мере накопления данных цели и задачи, для решения которых разрабатывается установка, были расширены. На основе представительной серии проведённых испытаний было принято решение анализировать полученные данные не только со статистической точки зрения (для эффекта Кайзера нужен лишь подсчет количества событий и анализ скорости роста их количества), но также оценить качественный состав сигналов АЭ, ответив на следующие вопросы:

- Что представляет собой единичный сигнал?

- Какой он имеет типичный спектр?

- На сколько спектр ограничен сверху по частоте? Хватает ли полосы пропускания оборудования для регистрации?

Для этого нами были отобраны более 50 цилиндрических образцов керна длиной 60 мм и 30 мм в диаметре разных литотипов: алевролиты, аргиллиты, песчаники с карбонатным цементом и среднезернистые песчаники с глинистым связующим. Образцы керна подвергались осевой нагрузке: в течение 50-150 секунд усилие на оси поднималось до 40-200 МПа до их полного разрушения. В ходе каждого эксперимента в двух каналах происходила непрерывная регистрация сигналов АЭ с пьезоэлектрических преобразователей, находящихся максимально близко к керну на его торцах. 
Эксперименты показали, что сигналы АЭ достаточно уверенно регистрируются даже имеющейся системой наблюдений, состоящей из 2-х датчиков, установленных по торцам керна, и аналого-цифрового преобразователя (АЦП) с частотой дискретизации 1 МГц включенного через предварительный усилитель. Усиления в 40 Дб так же оказалось вполне достаточно для достижения удовлетворительного соотношения сигнал/шум. На Рис.2 приведены типичный фильтрованный сигнал АЭ (а) и его спектр (б). Для удобства наглядного восприятия к исходному сигналу применен Фильтр Высоких Частот (ФВЧ) с нижней частотой 50 кГц. На графике видно, что верхняя частота сигнала АЭ при регистрации системой УИДК простирается значительно выше предполагаемого изначально значения в 100 кГц. Были зарегистрированы сигналы с частотой до 300 кГц, и, скорее всего, даже частоты дискретизации текущего АЦП не достаточно, чтобы увидеть весь интересующий сигнал. Это предположение подтверждается примерами сигналов и их спектров из программы InSite, в которых полоса частот ограничена примерно 1 МГц. (Рис. 2в). Есть так же данные, что в плотных горных породах, таких, например, как гранит, спектр сигналов АЭ содержит ещё более высокочастотные компоненты до 2 МГц.

Собранная информация о частотном составе сигналов АЭ является крайне важным параметром для разработки системы наблюдений и должна быть учтена при проектировании новых датчиков и новой системы регистрации данных. Так, из анализа полученных данных уже можно сделать вывод о том, что даже на керне относительно небольшого диаметра в 30 мм можно пытаться восстанавливать координаты событий АЭ. Заметим, что до проведения описанных экспериментов мы предполагали, что надёжная локализация событий АЭ возможна только на кернах диаметром как минимум 100 мм. Однако установки, работающие с керном большого диаметра, достаточно редкие и дорогие. Исследования же на керне малого диаметра широко распространены, имеется доступное геомеханическое оборудование для проведения одноосного и трехосного нагружения.

Стандартное лабораторное оборудование для измерения времени прохождения акустических волн через керн в пластовых условиях обычно содержит излучатель и приёмник ультразвуковых волн, обеспечивающие стабильную регистрацию на частотах вплоть до 0.5 МГц (см. Рис. 2б). Следовательно, имеющееся оборудование, состоящее из гидравлического пресса, системы нагружения и поддержания горного и обжимного давления вместе с акустическими датчиками может быть использовано для регистрации сигналов акустической эмиссии, возникающих в изучаемом образце горной породы при его нагружении в ячейке пресса. Более того, как показывают данные, представленные в описании программного обеспечения InSite, верхний диапазон частот допускает надёжное расширение до 2 МГц (Рис.2в).

\section{ЧИСЛЕННОЕ МОДЕЛИРОВАНИЕ АКУСТИЧЕСКОЙ ЭМИССИИ}

Моделирование АЭ позволяет проводить виртуальные эксперименты с цифровой моделью керна. Оно включает в себя этапы имитации возникновения источников АЭ в образцах керна, моделирование возникающего волнового поля и его запись на 
виртуальных датчиках, восстановление источников АЭ внутри керна путём решения обратной задачи. Результаты численного моделирования крайне важны для выбора оптимальной конфигурации многоканальной системы сбора данных, в том числе и определения необходимого количества каналов регистрации и положения датчиков относительно образца.

Цилиндрическая форма изучаемых образцов и стремление максимально точно учитывать их границы при численном моделировании диктуют необходимость его проведения либо в цилиндрической (3D), либо полярной (2D) системах координат. Их характерной особенностью является линейное разрастание азимутального размера ячейки с удалением от начала координат, что приводит к ухудшению качества пространственной аппроксимации. Поэтому, чтобы обеспечить желаемый порядок пространственной аппроксимации во всей области, мы используем цилиндрическую/полярную сетку с периодическим измельчением азимутального шага [Pissarenko et al., 2009]. Это позволяет обеспечить примерно одинаковый азимутальный и радиальный размер ячейки и тем самым добиться равномерной пространственной аппроксимации во всей расчётной области (Рис.3). При таком подходе, однако, возникает проблема согласования смежных сеток в окрестности цилиндров/окружностей, при переходе через которые производится периодическое азимутальное измельчение. Для того чтобы сохранить порядок аппроксимации исходной задачи, интерполяция данных с одной сетки на другую должна быть задана, как минимум, на порядок выше по точности, чем сама разностная схема. Кроме того, эта интерполяция должна выполняться на каждом шаге по времени, что накладывает весьма жесткие требования к быстродействию при её выполнении. Наиболее подходящей для данного случая является интерполяция с использованием быстрого преобразования Фурье (БПФ) [Pissarenko et al., 2009; Костин и др., 2008], которая и используется в нашем численном алгоритме для моделирования акустической эмиссии на керне.

Для изучения основных особенностей процессов акустической эмиссии и выполняемого в последствии продолжения в обратном времени, мы решили первоначально сосредоточиться на двумерном случае, чтобы избежать проведения весьма дорогих вычислений, необходимых в трёхмерном случае. При этом, при формировании модели и параметров экспериментов мы использовали геометрические параметры максимально приближённые к использующимся в лабораторных экспериментах.

В качестве определяющих уравнений использовались уравнения динамической теории упругости в полярной системе координат $(r, \theta)$ :

1. Уравнения движения

$$
\begin{aligned}
& \rho \frac{\partial u_{r}}{\partial t}=\frac{\partial \sigma_{r r}}{\partial r}+\frac{1}{r} \frac{\partial \sigma_{r \theta}}{\partial \theta}+\frac{1}{r}\left(\sigma_{r r}-\sigma_{\theta \theta}\right), \\
& \rho \frac{\partial u_{\theta}}{\partial t}=\frac{\partial \sigma_{r \theta}}{\partial r}+\frac{1}{r} \frac{\partial \sigma_{\theta \theta}}{\partial \theta}+\frac{2 \sigma_{r \theta}}{r} .
\end{aligned}
$$

2. Закон Гука (уравнения состояния) 


$$
\begin{aligned}
& \frac{\partial \sigma_{r r}}{\partial t}=(\lambda+2 \mu) \frac{\partial u_{r}}{\partial r}+\frac{\lambda}{r}\left(u_{r}+\frac{\partial u_{\theta}}{\partial \theta}\right)+F_{r}, \\
& \frac{\partial \sigma_{\theta \theta}}{\partial t}=\frac{(\lambda+2 \mu)}{r}\left(u_{r}+\frac{\partial u_{\theta}}{\partial \theta}\right)+\lambda \frac{\partial u_{r}}{\partial r}+F_{\theta}, \\
& \frac{\partial \sigma_{r \theta}}{\partial t}=\mu \frac{\partial u_{\theta}}{\partial r}+\frac{\mu}{r}\left(\frac{\partial u_{r}}{\partial \theta}-u_{\theta}\right) .
\end{aligned}
$$

3. Начальные условия

$$
\left.u_{r}\right|_{t=0}=\left.u_{\theta}\right|_{t=0}=\left.\sigma_{r r}\right|_{t=0}=\left.\sigma_{\theta \theta}\right|_{t=0}=\left.\sigma_{r \theta}\right|_{t=0}=0 .
$$

Система уравнений первого порядка (1-3) описывает распространение упругих колебаний в терминах компонент скоростей радиального $u_{r}$ и азимутального $u_{\theta}$ смещений, а так же компонент тензора напряжений $\sigma_{r r}, \sigma_{\theta \theta}, \sigma_{r \theta}$. Материальные параметры среды задаются модулями упругости Ламе $\lambda, \mu$ и плотностью $\rho$. В качестве граничных условий используется идеально-согласованный поглощающий граничный слой в виде в виде PML [Pissarenko et al, 2009; Костин и др., 2008].

Правые части в уравнениях (2) позволяют задавать источники разного типа. Чтобы задать точечный источник в виде центра расширения в точке $\left(r_{0}, \theta_{0}\right)$, достаточно положить

$$
F_{r}=F_{\theta}=f(t) \frac{\delta\left(r-r_{0}, \theta-\theta_{0}\right)}{2 \pi r},
$$

где $\delta$ - дельта-функция и $f(t)$ временной сигнал в источнике. Для того чтобы задать источник типа направленной силы, достаточно задать равной нулю одну из компонент $F_{r}$ или $F_{\theta}$. Так в случае $F_{r}=0$ получаем источник направленной силы в азимутальном направлении, а в случае $F_{\theta}=0$ - источник направленной силы в радиальном направлении.

На текущей стадии выполнения работ по численному моделированию АЭ мы не интересуемся физическими механизмами, приводящими к её возникновению. В численных расчетах события АЭ моделируются путем задания совокупности объемных источников, определяемых правыми частями $F=\left(F_{r}, F_{\theta}\right)$ уравнений (2). В приведенных ниже расчетах используется набор из трех точечных источников типа объемного расширения. Для заданных правых частей рассчитывается полное упругое волновое поле, которое регистрируется в некоторых точках (приемниках или каналах регистрации) на внешней окружности, охватывающей изучаемый образец.

Для численных экспериментов был выбран цилиндрический образец стандартного диаметра 30 миллиметров. Его упругие свойства задавались исходя из осреднённых свойств песчаника: $V_{p}=3500 \mathrm{~m} / \mathrm{c}, \quad V_{s}=2000 \mathrm{~m} / \mathrm{c} \quad$ и плотность $\rho=3500 \quad \mathrm{\kappa г} / \mathrm{m}^{3}$. Предполагалось, что источники сейсмоакустических волн располагаются в трех точках расчетной области и срабатывают в различные моменты времени: первый источник 
располагается в точке $\left(r_{81}, \theta_{01}=0\right)$ и срабатывает в момент точке $\left(r_{02}=10, \theta_{02}=\pi / 2\right)$ и срабатывает в момент $t_{02} \in 20 \quad$ и, наконец, третий в точке $\left(r_{03}\right.$, $\left.\theta_{03}=5 \pi / 4\right)$ и срабатывает в момент tөдкє30 . Правая часть (2) задавалась формулой

$$
F_{r}=F_{\theta}=f(t) \sum_{i=1}^{3} \frac{\delta\left(r-r_{0 i}, \theta-\theta_{0 i}\right)}{2 \pi r} .
$$

В качестве временного сигнала использовался Гауссиан $f(t)=\exp \left(-\left(\pi f_{0}\left(t-t_{0 i}\right)\right)^{2}\right)$, где $f_{0}$-доминирующая частота и $t_{0 i}$ - задержка импульса для $i$-го источника. С помощью выбора различных значений $t_{0 i}$ для каждого источника мы моделируем срабатывание источников в различные моменты времени. Время регистрации сигнала в приёмниках полагалось равным Тлғ40 . Приёмники равномерно располагаются на окружности, их номера отсчитываются от угла $\theta=0$ против часовой стрелки (Рис.4).

Для численного моделирования волнового поля, возбуждаемого источниками в образце керна, использовался явный конечно-разностный метод. Для аппроксимации уравнений системы (1-3) использовалась конечно-разностная схема со вторым порядком точности в узлах сдвинутой полярной сетки с периодическим азимутальным измельчением. Мы не останавливаемся на описании самого численного метода решения системы (1-3), он достаточно подобно изложен в работах [Pissarenko et al, 2009; Костин и др.; 2008]. Рассчитанное суммарное волновое поле регистрировалось в заданных приемниках (Рис. 5а). В численных экспериментах мы варьировали как число приёмников, так и доминирующую частоту импульса в источнике. Полученный синтетический набор данных затем использовался для отладки программного обеспечения, реализующего метод зеркального обращения и определения оптимальных параметров системы наблюдения.

\section{ЧИСЛЕННАЯ РЕАЛИЗАЦИЯ МЕТОДА ЗЕРКАЛЬНОГО ОБРАЩЕНИЯ ВРЕМЕНИ}

На этапе решения задачи по восстановлению событий АЭ мы «забываем» про расположение источников и времена их включения. Для их определения мы решаем обратную задачу путём продолжения зарегистрированных данных в обратном времени, который ниже будем называть Метод Зеркального Обращения Времени. Заметим, что в англоязычной литературе он имеет устоявшееся название Time Reversal Mirror (TRM) [Prada and Fink, 1998; Fink and Prada, 2001; José, Yuanwei, 2007; Parvasi et al., 2016; Fink, 2002, 2008]. Остановимся кратко на его описании.

Метод зеркального обращения времени опирается на один из классических принципов современной физики - принцип обратимости времени для линейных процессов без затухания. В последнее время этот метод получил распространение как способ дефектоскопии для широкого спектра исследований, связанных с тестированием различных технических устройств [Griffa et al., 2008; Lints et al., 2020]. В настоящей 
работе этот принцип применяется к лабораторным исследованиям керна, а именно к процессу сейсмической эмиссии, возникающей при нагружении образцов керна. Её источники связаны с наличием в образцах микронарушений, таких, например, как микротрещины, неоднородные зоны со скоплениями материалов с различными механическими свойствами и др. Естественно допустить, что именно в таких зонах будут развиваться нарушения целостности горной породы, например, при выполнении гидроразрыва пласта.

Напомним, что базовый эксперимент метода зеркального времени заключается в записи колебаний, вызванных действием точечного источника на поверхности $S$, охватывающей источник и некоторый объём пространства $V$, его окружающий. Принципиальным здесь является тот факт, что на поверхности $\mathrm{S}$ не ставится никаких граничных условий. Поэтому в численных экспериментах мы считаем, что данные регистрируются в «свободном» пространстве. Для этого вся расчётная область окружается идеально-согласованным поглощающим граничным слоем в виде (PML - Perfectly Matched Layer) в модификации для цилиндрической системы координат [Pissarenko et al, 2009; Костин и др., 2008]. Заметим, что эти поглощающие условия хорошо согласуются с конструкцией оборудования, а именно - наличием демпфирующей манжеты, охватывающей образец. Таким образом, используемая нами схема проведения численного эксперимента, представленная на Рис.4, полностью удовлетворяет требованиям к эксперименту по методу зеркального обращения времени, сформулированным в работах [Fink and Prada, 2001; Givoli, 2014].

Итак, обратная задача заключается в восстановлении положений источников и времён их включения по записям волновых полей в наборе приемников. Для ее решения поступаем следующим образом. Сначала меняем времена трасс на противоположные, «переворачивая» их по времени (Рис. 5б). Затем «превращаем» каждый приёмник в источник, излучающий «перевернутую» трассу. Для этого в правую часть уравнений динамической теории упругости (2) помещаем точечные источники, координаты которых совпадают с координатами приёмников, а излучаемый сигнал есть обращённая во времени запись. После чего рассчитывается волновое поле в обратном времени, то есть от момента окончания записи Т до нуля. Заметим, что эта операция является корректной, как задача Коши для волнового уравнения, в силу принципа обратимости во времени линейных волновых процессов без затухания.

На Рис.6 и Рис.7 представлены моментальные снимки волнового поля в обратном времени для компоненты $u_{r}$ в различные моменты времени, соответствующие временам срабатывания источников АЭ. На Рис.6 расчеты приведены для случая $f_{0}=1$ МГц и 24 приемников/источников на внешней окружности образца, а на Рис.7 для случая $f_{0}=250$ кГц и 8 приемников/источников. Можно заметить локальные разрастания амплитуд в местах расположения источников АЭ. Однако уверенно выделить эти события на фоне сложной волновой картины довольно затруднительно из-за множества локальных максимумов и минимумов.

Для того, чтобы усилить когерентную составляющую рассчитанного в обратном времени полного волнового поля, мы перешли к вычислению «накопленной» упругой 
энергии. Для этого в каждый момент времени $t_{k}$ для всех точек конечно-разностной сетки $\left(r_{i}, \theta_{j}\right)$ рассчитывается квадрат суммарной энергии $E$ для всех предыдущих шагов $t_{m}$ по времени:

$$
\begin{gathered}
E\left(r_{i}, \theta_{j}, t_{k}\right)=\sum_{t_{m} \unlhd_{k}} \sigma_{r r}\left(r_{i}, \theta_{j}, t_{m}\right) \varepsilon_{r r}\left(r_{i}, \theta_{j}, t_{m}\right)+\sigma_{\theta \theta}\left(r_{i}, \theta_{j}, t_{m}\right) \varepsilon_{\theta \theta}\left(r_{i}, \theta_{j}, t_{m}\right) \\
+2 \sigma_{r \theta}\left(r_{i}, \theta_{j}, t_{m}\right) \varepsilon_{r \theta}\left(r_{i}, \theta_{j}, t_{m}\right)
\end{gathered}
$$

На Рис.9 приведены снимки волнового поля суммарной энергии, рассчитанные по формуле (6) для времен, соответствующих моментам включения первого второго и третьего источников для различных значений доминирующей частоты в источнике. Выбор частот производился, исходя из анализа записей волнового поля для реальных лабораторных экспериментов (Рис.2). Как видно из Рис.2а на имеющемся оборудовании УИДК удаётся получить сигналы, на которых уверенно наблюдаются частоты 200 - 300 кГц. Поэтому представленные ниже численные расчёты начинаются с частоты 250 кГц. В то же время, как показано на Рис. 2б, использование другого оборудования обеспечивает наличие в сигнале полезной информации вплоть до частот в 1,5 МГц. Поэтому мы решили провести вычисления также для частот 500 кГц и 1 МГц.

Ещё одним принципиальным моментом при планировании экспериментов является число приёмников/источников (вторичных), охватывающих область наблюдения. Для образцов керна диаметром в 30 мм, длина окружности, на которой нужно разместить требуемое количество приёмников, составляет примерно 100 мм. Естественно, что наряду с доминирующей частотой число приёмников играет определяющую роль в качестве восстановления расположения и времени включения источников АЭ. Поэтому в наших численных экспериментах были построены изображения для различного числа приёмников, охватывающих исследуемый образец керна: 24,12 , и 6 . Полученные при этом изображения представлены на Рис. 8 - 10. Обратим внимание на появление артефактов на изображениях, связанных с наличием приёмников, которые при обращении волнового поля в обратном времени превращаются в источники. Их появление объясняется тем фактом, что при численном моделировании любой источник приводит к возникновению особенности волнового поля в его окрестности. Именно эта особенность и проявляется в виде артефакта на изображении продолженного в обратном времени волнового поля. В связи с тем, что действие этих источников весьма длительно по времени, их проявление в обращённых волновых полях прослеживается практически на всём временном интервале. Однако их пространственное положение нам известно абсолютно точно, так как совпадает с известным расположением приёмников. Следовательно, мы легко можем идентифицировать эти разрастания амплитуды, как артефакты, не связанные с событиями АЭ.

Первая серия расчётов выполнялась для 24 приёмников для набора доминирующих частот 250 кГц, 500 кГц и 1 МГц. Результаты моделирования приведены на Рисунке 8. Для облегчения анализа, мы приводим пространственное распределение сейсмоакустической энергии только для моментов времени, соответствующих включению источников. Как видно, на полученных изображениях в каждый момент времени присутствуют только те источники, которые либо уже сработали, либо включились в данный момент времени. Как и следовало ожидать, разрешающая способность, которая здесь обратно пропорциональна 
ширине полученного изображения, увеличивается при возрастании доминирующей частоты. Здесь нужно обратить внимание на тот факт, что на полученных изображениях практически не проявляется наличие источников-приёмников, излучающих сигнал в обратном времени, кроме момента времени t2, соответствующего включению второго источника. Этот факт объясняется тем, что второй источник расположен ближе всего к одному из источников-приёмников, размещённых на внешней окружности.

Как уже было отмечено, приёмники, которые используются для продолжения волнового поля в обратном времени, располагаются на внешней стороне керна диаметром 30 мм и длиной окружности равной примерно 100 мм. Естественно, что на ней вряд ли удастся разместить 24 источника, так как в этом случае расстояние между ними будет примерно 4 мм. Поэтому следующая серия численных экспериментов была направлена на изучения качества локализации источников и определения моментов их включения при уменьшении количества регистрирующих приёмников, то есть увеличения расстояния между ними.

На Рисунке 9 приведены результаты численных экспериментов с использованием 12 приёмников. В этом случае расстояние между ними уже составляет примерно 1 см. Как видно, разрешающая способность, которая характеризуется размером получаемого изображения для единичного источника, остаётся примерно той же. В тоже время, заметно усиливается интенсивность артефактов, связанных с приёмниками-источниками, расположенными на внешнем диаметре изучаемого керна. Интересно отметить, что эти артефакты начинают появляться в момент включения источника и они тем меньше, чем выше доминирующая частота. Также отметим, что наиболее заметный артефакт связан с приёмником-источником, наиболее близким к источнику внутри керна. Вновь качество изображения заметно улучшается в смысле уменьшения интенсивности артефактов, при увеличении частоты.

Заключительная серия численных экспериментов проведена для шести приёмников и представлена на Рисунке 10. В этом случае расстояние между приёмниками равно примерно 1.5 см, что уже примерно соответствует реальной системе регистрации.

Видно, что поведение полученных изображений принципиально не отличается от двух предыдущих, полученных для 24 и 12 приёмников. Как и следовало ожидать, интенсивность артефактов здесь становится значительно выше. Однако, как и в предыдущих расчётах они локализованы в приёмниках-источниках и, следовательно, могут быть легко отделены от изображения физически существующих источников АЭ.

Для проверки численной устойчивости предложенной методики по отношению к шумам в данных и другим помехам была проведена серия дополнительных тестов. Для этого в сейсмотрассы приемников добавлялся 10-20\% «белый шум» с помощью генератора случайных величин с гауссовским распределением с нулевым средним и единичным отклонением. Полученные изображения практически не отличаются от тех, что представлены на Рис. 8-10. Для сравнения, приведем лишь один моментальный снимок суммарной энергии обращённого волнового поля, вычисленный при добавлении 10\% шума для частоты 250 кГц, 6 приёмников и момента времени t1=30 мкс (Рис. 11).

\section{ЗАКЛЮЧЕНИЕ}


В работе представлены результаты численных экспериментов по локализации и определения момента «включения» источников акустической эмиссии в образцах керна. Для этого использовался метод зеркального обращения времени, при котором приёмники, регистрирующие акустическую эмиссию, выступают впоследствии как источники, излучающие записанное волновое поле в обратном времени, то есть от финального времени записи $\mathrm{t}=\mathrm{T}$ до начального момента времени $\mathrm{t}=0$.

Как показали выполненные численные эксперименты, такая процедура позволяет не только локализовать источники акустической эмиссии, но и определить их время «включения». Была исследована разрешающая способность метода для набора частот и разного количества приемников/каналов регистрации. Было показано, что даже для частот порядка 250-500 кГц удается локализовать события АЭ. Важной особенностью предложенной методики является её устойчивость по отношению к шумам в данных и другим помехам, поскольку математическая задача сводится к решению задачи Коши для классической гиперболической системы динамической теории упругости. Как известно, такая задача корректна по Адамару и, следовательно, малые возмущения входных данных порождают малые возмущения решения.

В заключение отметим, что нашей ближайшей целью является проведение аналогичных расчётов в трёхмерном случае. При этом одной из основных целей будет определение оптимальной геометрии приёмников. Предварительно здесь нам видится использование либо кольцевых, либо спиральных структур. Окончательный ответ и предполагается получить путём проведения численных экспериментов.

\section{БЛАГОДАРНОСТИ}

Исследование выполнено при финансовой поддержке РФФИ в рамках научного проекта № 19-01-00347 А.

\section{БИБЛИОГРАФИЧЕСКИЙ СПИСОК}

Дрягин В.В., Володин И.А., Чеботарева И.Я. Акустические эффекты при деформировании структурно неоднородных сред // Акустический журнал . - 2017 . -№1 . - С. 84-93.

Иванов В.И., Барат В.А. Акустико-эмиссионная диагностика. М.: Спектр, 2017. - 362 с.

Иголкина Г.В., Дрягин В.В. Использование каротажа сейсмоакустической эмиссии для выделения флюидонасыщенных коллекторов в нефтегазовых скважинах // Нефтепромысловое дело. - 2018. - № 3. - С. 43-49.

Костин В.И., Решетова Г.В., Чеверда В.А. Численное моделирование трёхмерного акустического каротажа с использованием многопроцессорных вычислительных систем // Математическое моделирование. - 2008. - Т. 20. № 9. - С. 51-66. 
Кочарян Г.Г., Остапчук А.А. Акустическая эмиссия при различных режимах межблоковых перемещений // Физико-технические проблемы разработки полезных ископаемых. - 2015. №1. - C. 4-14.

Кретов Е. Ультразвуковая дефектоскопия в энергомашиностроении. М.: СВЕН, 2014. - 312 c.

Шадрин А. В. Геофизический критерий предвыбросного развития трещин в угольном пласте // Физико-технические проблемы разработки полезных ископаемых. - 2016. - № 4. C. 48-62.

Шайко-Шайковский А. Г., Богорош А. Т., Воронов С. А., Марченко К. В. Обзор применения акустической эмиссии для выявления микро- и нанодефектов // Надежность и качество сложных систем. - 2016. - № 1 (13). - С. 47-57.

Шкуратник В.Л., Новиков Е.А. Влияние механического нагружения каменной соли на параметры термостимулированной акустической эмиссии // Прикладная механика и техническая физика. - 2015. - Т. 56. № 3 (331). - С. 164-172.

Шкуратник В.Л., Филимонов Ю.Л., Кучурин С.В. Экспериментальные исследования акустической эмиссии в образцах угля при одноосном нагружении // Физико-технические проблемы разработки полезных ископаемых. -2004. - С. 42-49.

Щербаков И.П., Чмель А.Е. Конкурентное накопление повреждений внутри и между зерен при разрушении гранита при повышенных температурах // Геология и геофизика. - 2016. №11. - С. 151-158.

Bai X, Zhang D-m, Wang H, Li S-j, Rao Z. A novel in situ stress measurement method based on acoustic emission Kaiser effect: a theoretical and experimental study // R. Soc. open sci. 5: 181263. 2018. http://dx.doi.org/10.1098/rsos.181263

Givoli D. Time reversal as computational tool in acoustics and elastodynamics // Journal of Computational Acoustics. - 2014. - 22(3) - 1430001.

Fink, M. Acoustic Time-Reversal Mirrors // Topics Appl. Phys. - 2002. - 84. -P. 17-43.

Fink, M. Time-reversal acoustics // J. Phys.: Conf. Ser. - 2008. - 118(1), 012001.

Fink, M., and Prada, C. Acoustic time-reversal mirrors // Inv. Problems. - 2001. - 17(1), R1-R38.

Griffa M., Anderson B.E., Guyer R.A., Ulrich T.J., Robinson P.A. Investigation of the robustness of time reversal acoustics in solid media through the reconstruction of temporal symmetric sources // J. Phys. D: Appl. Phys. - 2008. - 41(8). - 085415.

Grosse, C., Ohtsu M. Acoustic Emission Testing: Basics for Research - Applications in Civil Engineering; With Contributions by Numerous Experts. Springer, Heidelberg, 2008. -406 p.

José M. F. M., Yuanwei J. Detection by Time Reversal: Single Antenna // IEEE Transactions on Signal Processing. - 2007. - 55(1). - P. 187-201.

Kaiser J. Untersuchung über das Auftreten von Geräuschen beim Zugversuch // Dr.-Ing. Dissertation, Fakultät für Maschinenwesen und Elektrotechnik der Technischen Universität München (TUM); 15.2.1950. 
Lints M., Salupere A., Dos Saqntos S. Numerical simulation of ultrasonic time reversal on defects in carbon fibre reinforced polymer // Wave motion. - 2020. - V.94. - 102526.

Ohtsu M. Acoustic Emission and Related Non-destructive Evaluation Techniques in the Fracture Mechanics of Concrete: Fundamentals and Applications. Woodhead Publishing Series in Civil and Structural Engineering, Elsevier Science, 2015. - 318 p.

Parvasi, S. M., Ho, Siu Chun M., Kong, Q., Mousavi, R., Song, G. Real time bolt preload monitoring using piezoceramic transducers and time reversal technique - a numerical study with experimental verification // Smart Materials and Structures. -2016. - 25(8).

Pissarenko, D.; Reshetova, G.V.; Tcheverda, V.A. 3D finite-difference synthetic acoustic logging in cylindrical coordinates // Geophysical Prospecting. - 2009. - 57(3). - P. 367-377.

Prada C., Fink M. Separation of interfering acoustic scattered signals using the invariants of the time reversal operator: application to Lamb waves characterization // J. Acoust. Soc. Am. - 1998. - 104(2). - P. 801-807. 
Рис.1. Установка УИДК для исследования прочностных свойств керна в пластовых условиях производства АО «Геологика».

Рис. 2. Фильтрованный сигнал АЭ ФВЧ 50 кГц (а) и его спектр (б). Спектр сигнала АЭ из примеров программы InSite (в).

Рис.3. Сетка с последовательным азимутальным измельчением.

Рис.4. Расположение источников (красный) и приёмников (черный) в численных экспериментах. Поглощающий граничный слой PML (серый).

Рис.5. Сейсмограмма, записанная для 24 приёмников, расположенных на окружности, охватывающей целевую область (а). Сейсмограмма, перевёрнутая по времени и используемая на втором этапе восстановления положений источников (б). Частота источников $f_{0}=1$ МГц.

Рис.6. Моментальные снимки волнового поля в обратном времени для компоненты $u_{r}$ в начальный момент (а), в момент фокусировки волнового поля в третьем источнике $\begin{array}{lll}t \lambda z k 60 & \text { (б), во втором источнике } t_{\text {s }} 20 & \text { (в) и в первом источнике } t_{\lambda \neq k 30}\end{array}$ Расчеты приведены для случая $f_{0}=1$ МГц и 24 приемников/источников на внешней окружности образца.

Рис.7. Моментальные снимки волнового поля в обратном времени для компоненты $u_{r}$ в начальный момент (а), в момент фокусировки волнового поля в третьем источнике

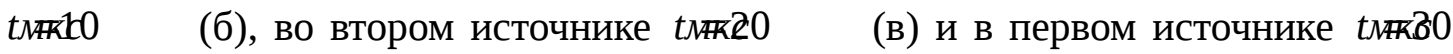
Расчеты приведены для случая $f_{0}=250$ кГц и 8 приемников/источников на внешней окружности образца.

Рис.8. Моментальные снимки суммарной энергии обращённого волнового поля для времён $\mathrm{t} 3=10$ мкс, t2=20 мкс, $\mathrm{t} 1=30$ мкс, соответствующих включению третьего, второго и первого источников, при использовании 24 приёмников.

Рис.9. Моментальные снимки суммарной энергии обращённого волнового поля для времён $\mathrm{t} 3=10$ мкс, $\mathrm{t} 2=20$ мкс, $\mathrm{t} 1=30$ мкс, соответствующих включению третьего, второго и первого источников, при использовании 12 приёмников.

Рис.10. Моментальные снимки суммарной энергии обращённого волнового поля для времён $\mathrm{t} 3=10$ мкс, $\mathrm{t} 2=20$ мкс, $\mathrm{t} 1=30$ мкс, соответствующих включению третьего, второго и первого источников, при использовании 6 приёмников.

Рис.11. Моментальный снимок суммарной энергии обращённого волнового поля, вычисленный при добавлении 10\% шума для частоты 250 кГц, 6 приёмников и момента времени $\mathrm{t} 1=30$ мкс. 


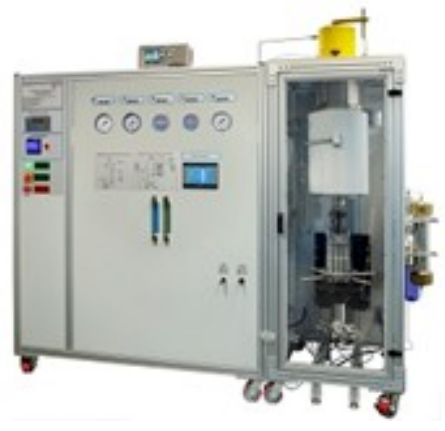




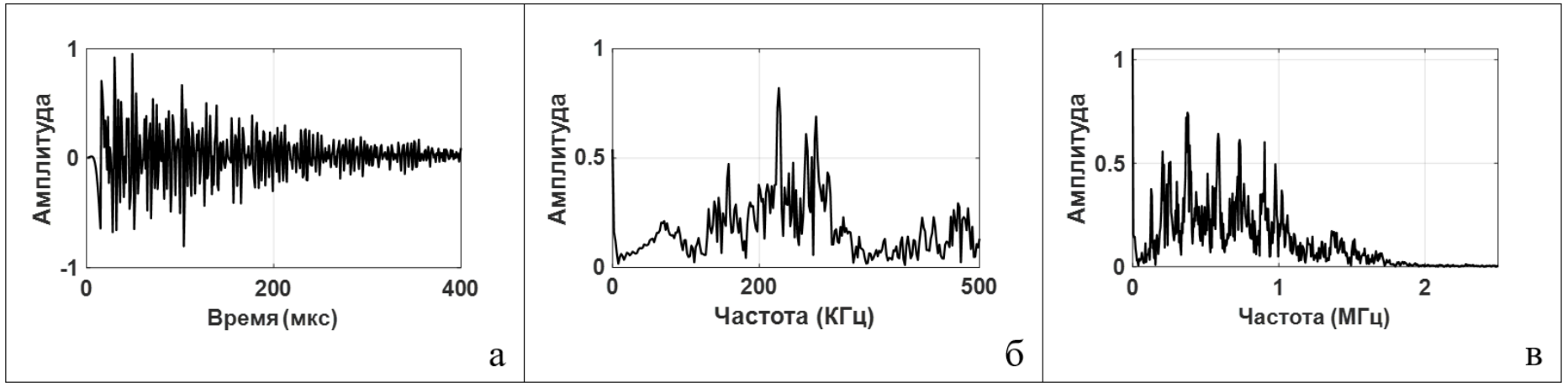




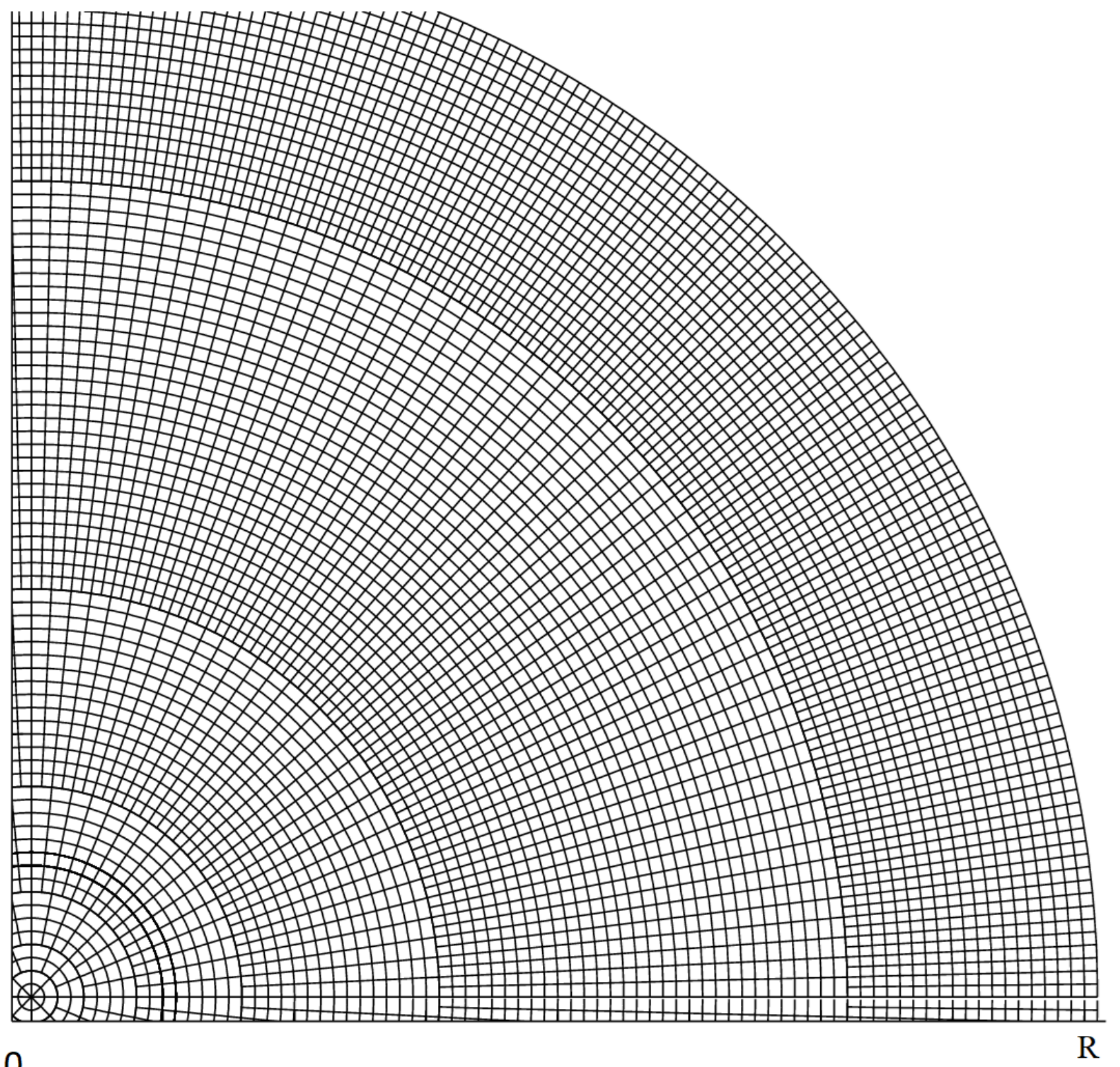




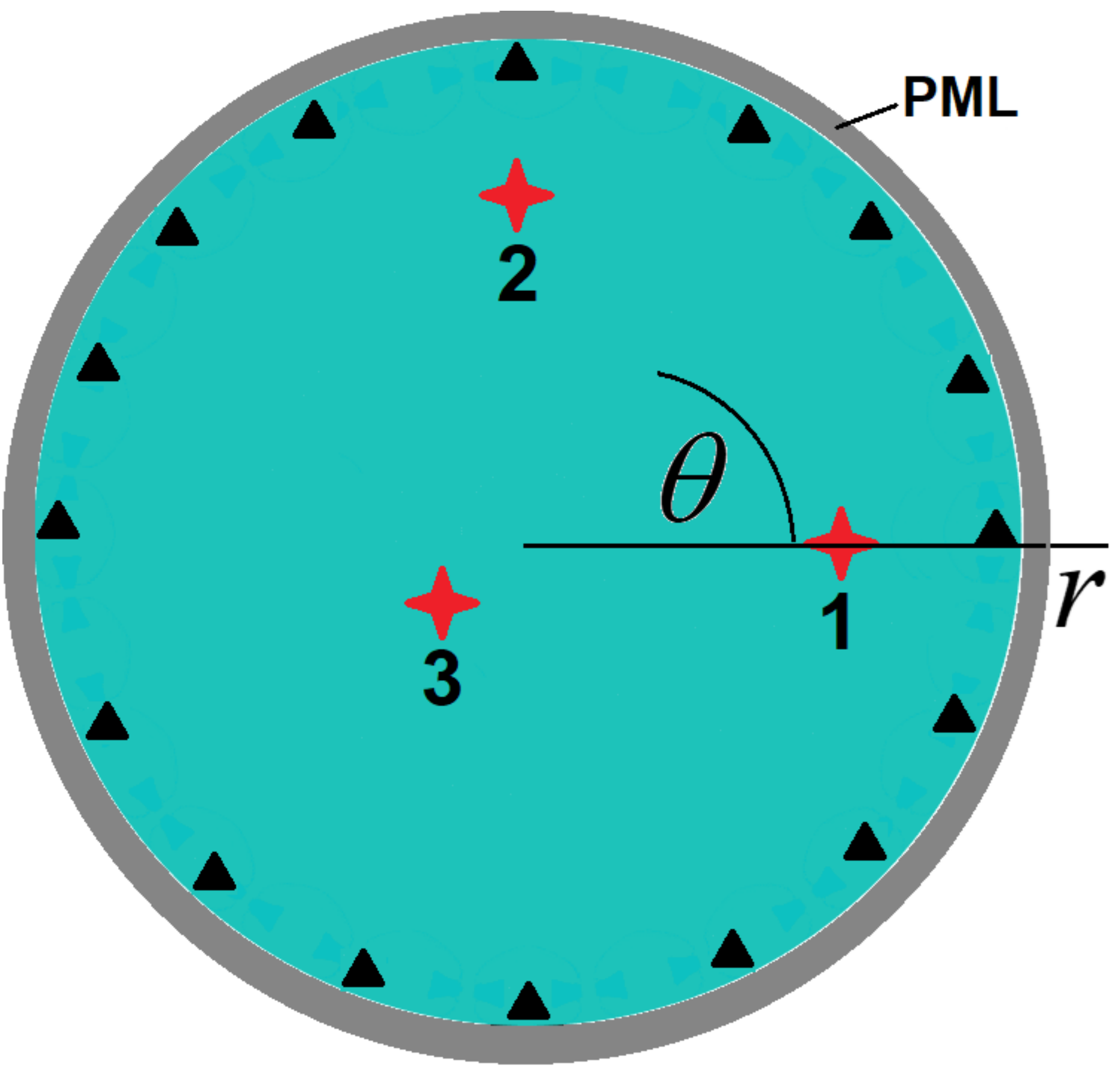




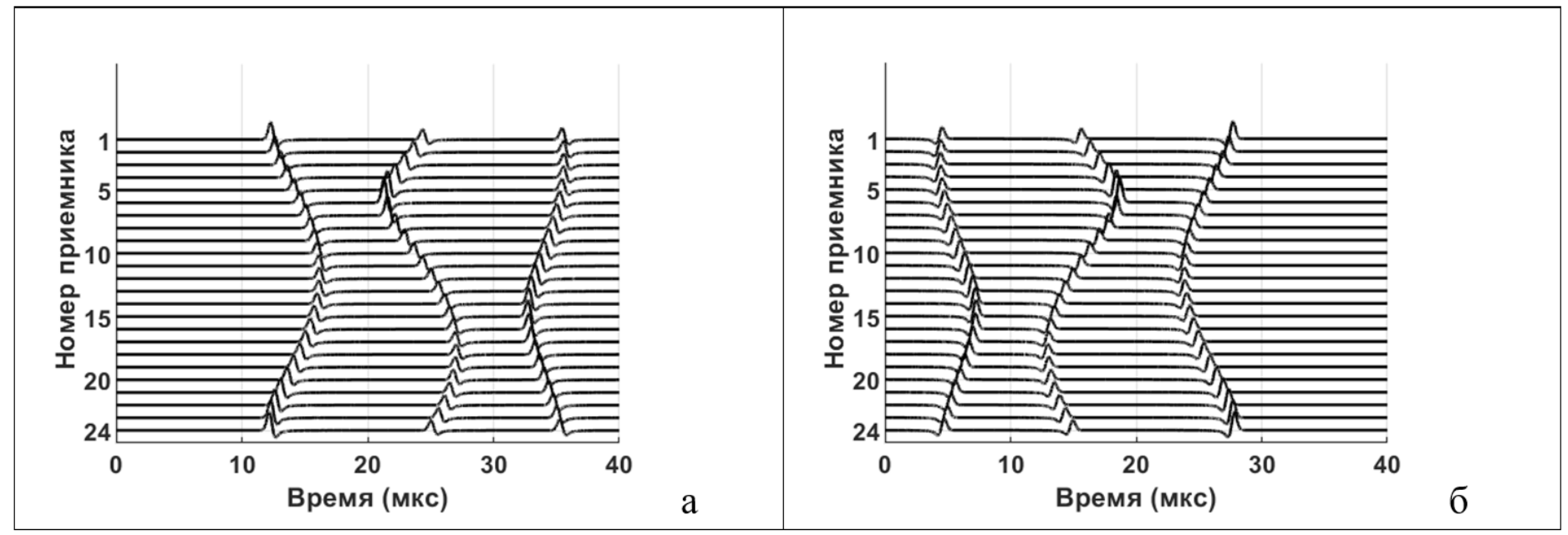




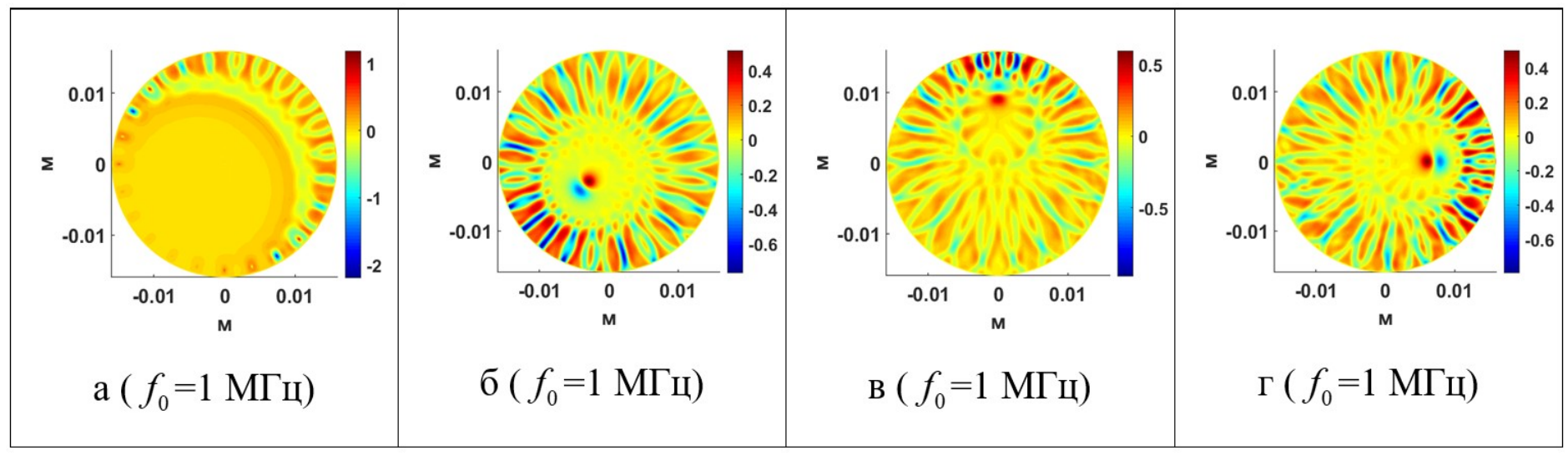




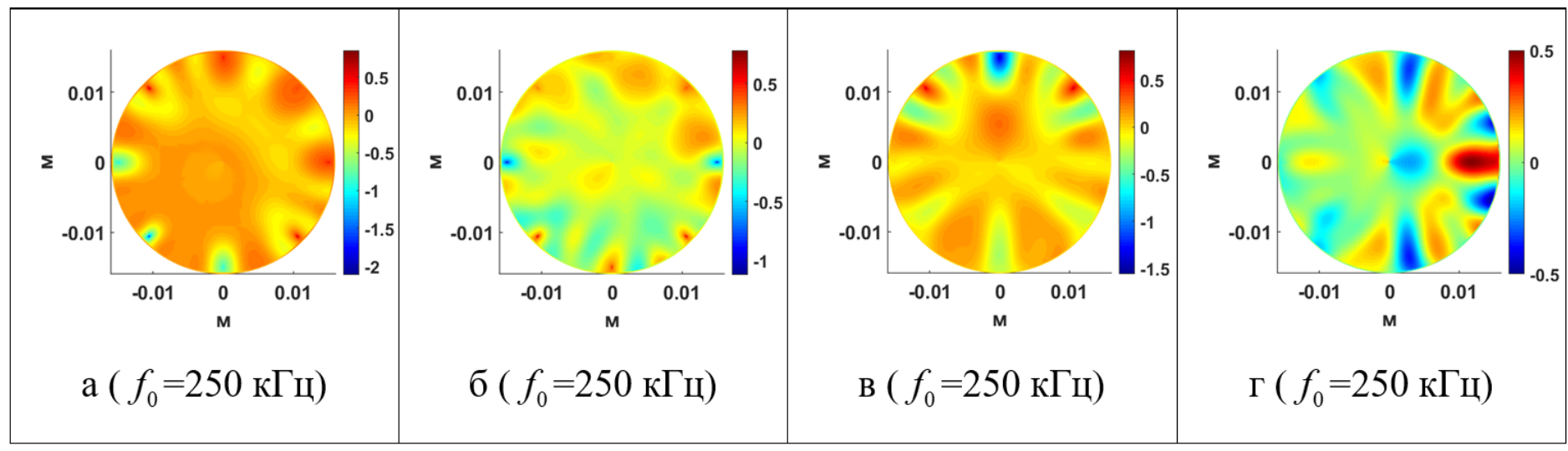




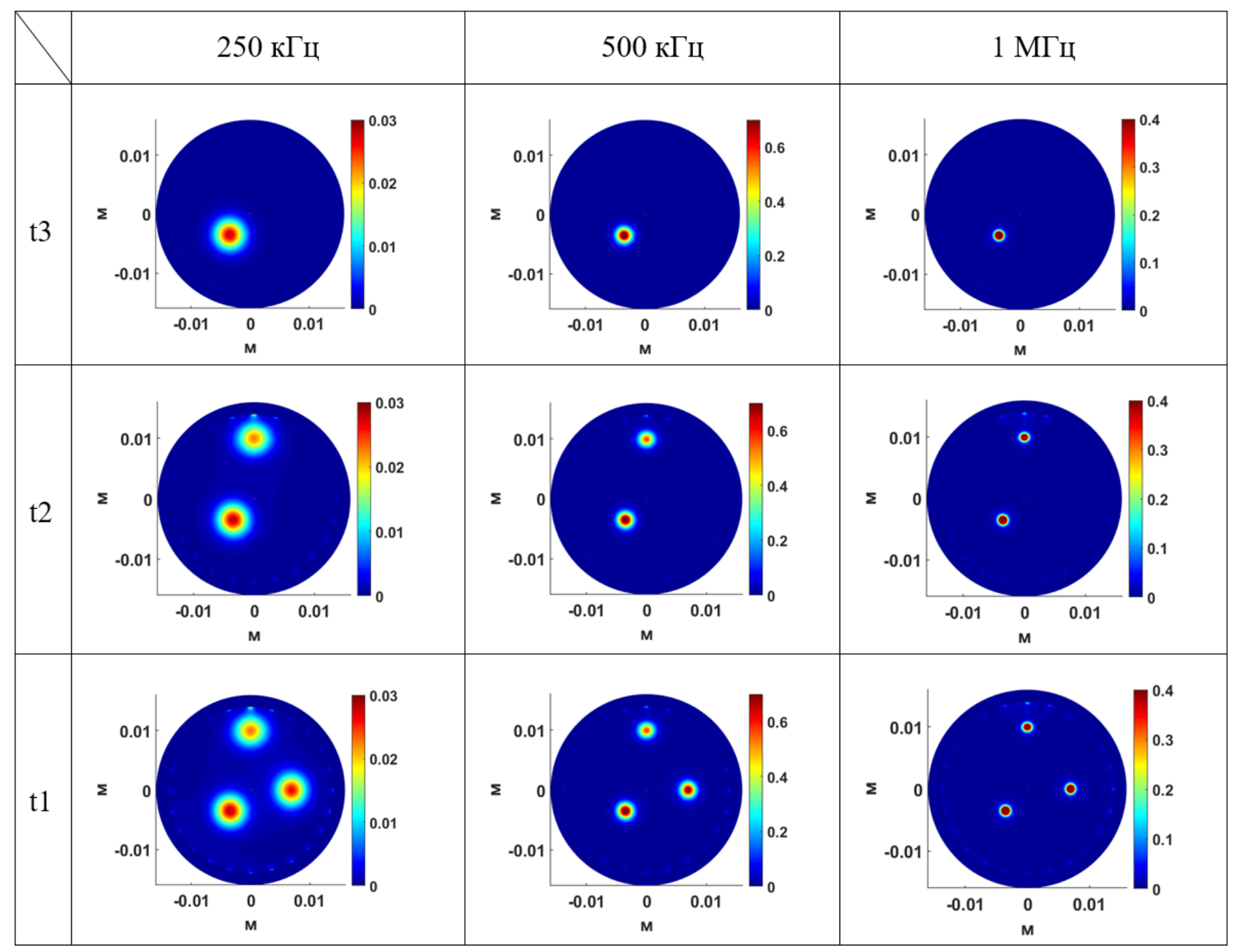




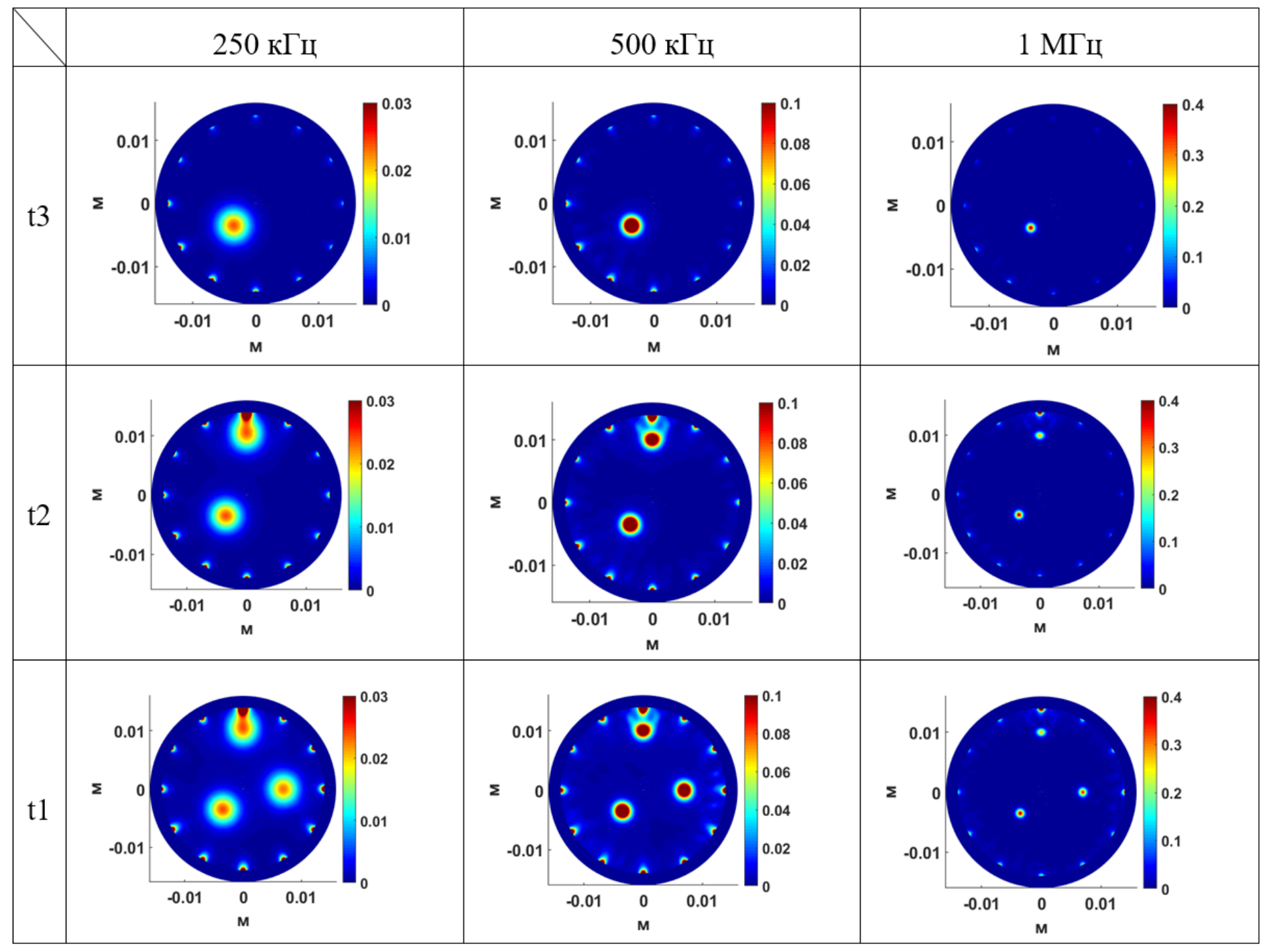




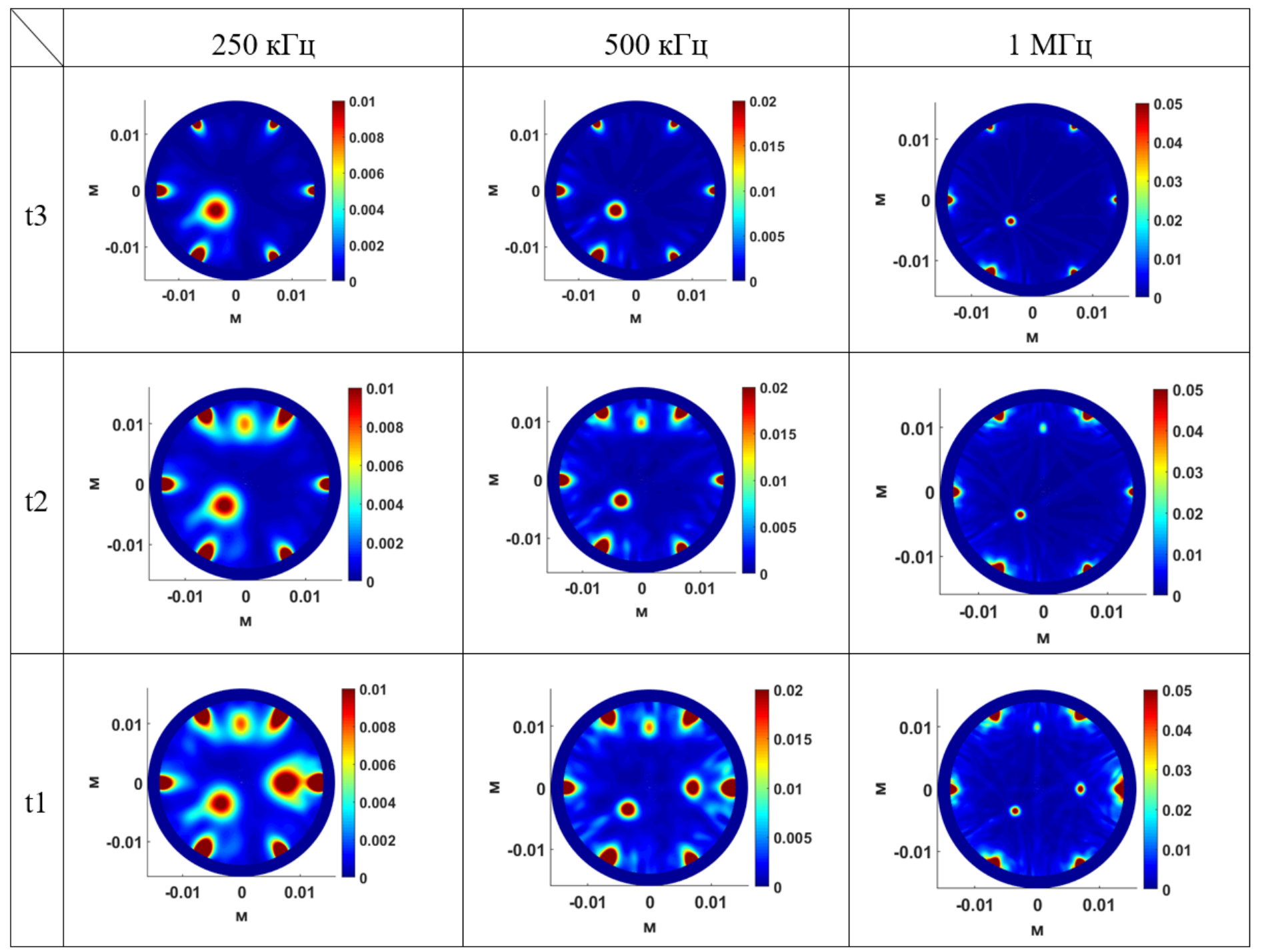




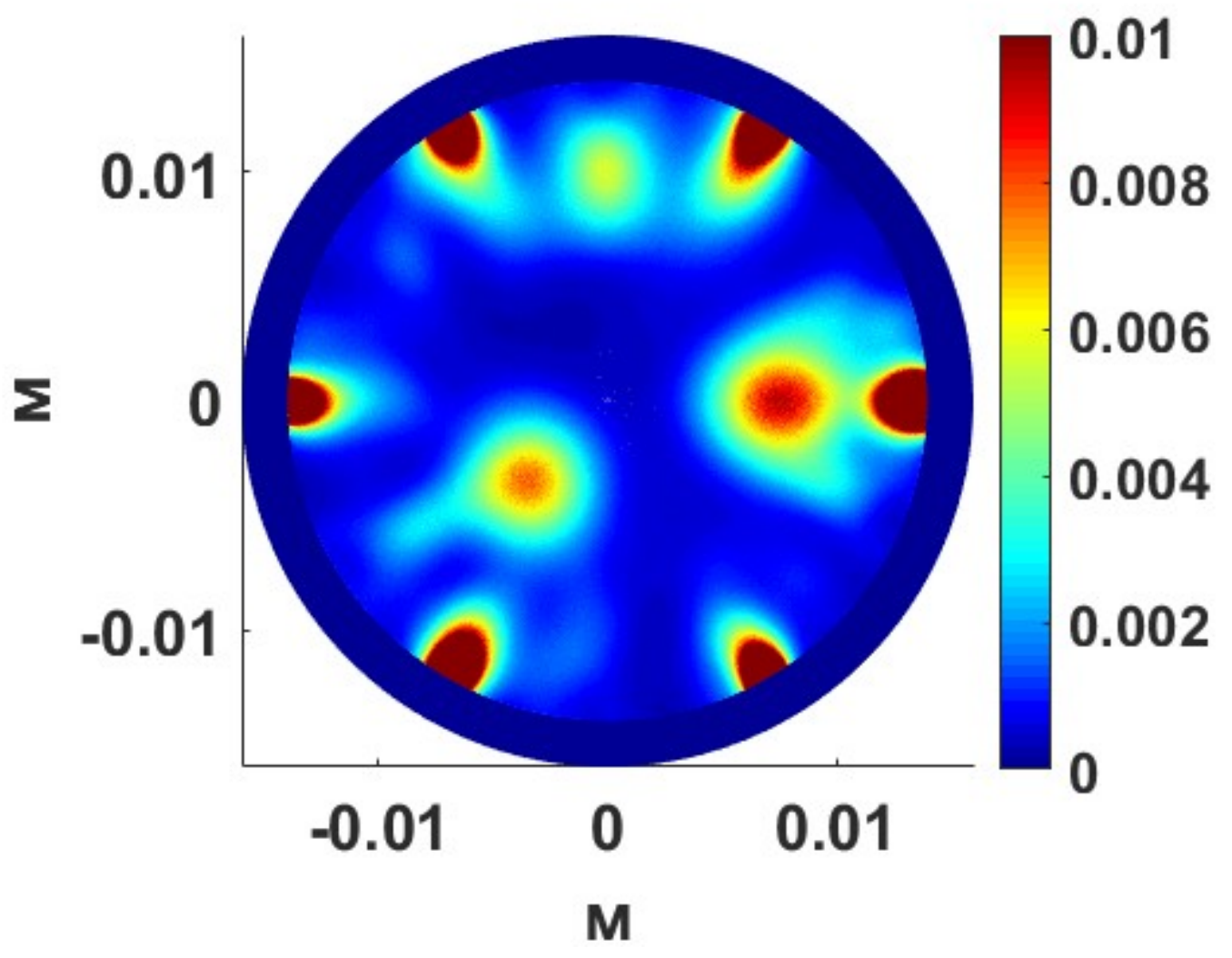

